BMC Bioinformatics

\title{
Base-pair ambiguity and the kinetics of RNA folding
}

\author{
Guangyao Zhou $^{1 *}$ (D) Jackson Loper² and Stuart Geman ${ }^{3}$
}

\begin{abstract}
Background: A folding RNA molecule encounters multiple opportunities to form non-native yet energetically favorable pairings of nucleotide sequences. Given this forbidding free-energy landscape, mechanisms have evolved that contribute to a directed and efficient folding process, including catalytic proteins and error-detecting chaperones. Among structural RNA molecules we make a distinction between "bound" molecules, which are active as part of ribonucleoprotein (RNP) complexes, and "unbound," with physiological functions performed without necessarily being bound in RNP complexes. We hypothesized that unbound molecules, lacking the partnering structure of a protein, would be more vulnerable than bound molecules to kinetic traps that compete with native stem structures. We defined an "ambiguity index" — a normalized function of the primary and secondary structure of an individual molecule that measures the number of kinetic traps available to nucleotide sequences that are paired in the native structure, presuming that unbound molecules would have lower indexes. The ambiguity index depends on the purported secondary structure, and was computed under both the comparative ("gold standard") and an equilibrium-based prediction which approximates the minimum free energy (MFE) structure. Arguing that kinetically accessible metastable structures might be more biologically relevant than thermodynamic equilibrium structures, we also hypothesized that MFE-derived ambiguities would be less effective in separating bound and unbound molecules.

Results: We have introduced an intuitive and easily computed function of primary and secondary structures that measures the availability of complementary sequences that could disrupt the formation of native stems on a given molecule —an ambiguity index. Using comparative secondary structures, the ambiguity index is systematically smaller among unbound than bound molecules, as expected. Furthermore, the effect is lost when the presumably more accurate comparative structure is replaced instead by the MFE structure.
\end{abstract}

Conclusions: A statistical analysis of the relationship between the primary and secondary structures of non-coding RNA molecules suggests that stem-disrupting kinetic traps are substantially less prevalent in molecules not participating in RNP complexes. In that this distinction is apparent under the comparative but not the MFE secondary structure, the results highlight a possible deficiency in structure predictions when based upon assumptions of thermodynamic equilibrium.

Keywords: Non-coding RNA, RNA folding kinetics, Comparative secondary structure, Minimum free energy, Thermodynamic equilibrium, Self-splicing introns, Ribonucleoproteins

\section{Background}

Discoveries in recent decades have established a wide range of biological roles served by RNA molecules, in addition to their better-known role as carriers of the coded messages that direct ribosomes to construct specific proteins. Non-coding RNA molecules participate

\footnotetext{
*Correspondence: tczhouguangyao@gmail.com

'Vicarious Al, Union City, CA USA

Full list of author information is available at the end of the article
}

in gene regulation, DNA and RNA repair, splicing and self-splicing, catalysis, protein synthesis, and intracellular transportation $[1,2]$. The precursors to these actions include a multitude of processes through which primary structures are transformed into stable or metastable secondary and tertiary structures. There are many gaps in our knowledge, but accumulating evidence (cf. [3-8]) suggests that the full story typically includes cotranscriptional explorations of secondary and tertiary structures, possibly

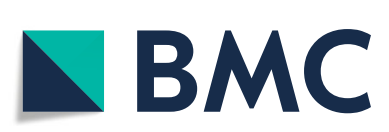

(c) The Author(s). 2019 Open Access This article is distributed under the terms of the Creative Commons Attribution 4.0 International License (http://creativecommons.org/licenses/by/4.0/), which permits unrestricted use, distribution, and reproduction in any medium, provided you give appropriate credit to the original author(s) and the source, provide a link to the Creative Commons license, and indicate if changes were made. The Creative Commons Public Domain Dedication waiver (http://creativecommons.org/publicdomain/zero/1.0/) applies to the data made available in this article, unless otherwise stated. 
accompanied by finely regulated transcription speeds, as well as a selection of proteins that may participate as stabilizers, catalysts, partners in a ribonculeoprotein complex, or chaperones to guide the process and detect errors. It is not surprising, then, that although many non-coding RNA molecules can be coxed into folding, properly, in artificial environments, the results rarely if ever match in vivo production in terms of speed or yield $[3,4,9,10]$.

Nevertheless, given the infamously rugged free-energy landscape of all but the smallest RNA molecules, there is good reason to expect that many of the large structural RNA molecules evolved not only towards a useful tertiary structure but also, at the same time, to help navigate the energy landscape. We reasoned that this process, a kind of co-evolution of pathway and structure, might have left a statistical signature, or "tell," in the relationships between primary and native secondary structures. The primary structures of RNA molecules typically afford many opportunities to form short or medium-length stems, ${ }^{1}$ most of which do not participate in the native structure. This not only makes it hard for the computational biologist to accurately predict secondary structure, but might equally challenge the biological process to avoid these kinetic traps. Once formed, they require a large amount of energy (not to mention time) to be unformed.

Taking this kinetic point of view a step further, we conjectured that evolutionary pressures would tend to suppress the relative prevalence of ambiguous pairings, meaning available complementary subsequences, more for those subsequences that include paired nucleotides in the native structure than for equally long subsequences that do not. The idea being that ambiguities of stemparticipating subsequences would directly compete with native stem formations and therefore be more likely to inhibit folding. Here, we do not mean to suggest that these particular adaptive mechanisms would obviate the need or advantages of other adaptations[3, 5, 11, 12], including the reliance on proteins as both nonspecific and specific cofactors. Herschlag [3] (and many others since) argued convincingly that thermodynamic considerations applied to an unaccompanied RNA molecule could explain neither the folding process nor the stability of the folded product, explicitly anticipating multiple roles for protein cofactors. It is by now apparent that many mechanisms have evolved, and are still evolving, to support repeatable and efficient RNA folding[3, 5, 11-15]. We are suggesting that some of these, perhaps among the earliest, might be visible upon close examination of relationships between the availability of ambiguous pairings for stem structures to those for non-stem structures. Shortly, we will introduce a formal definition of this relative ambiguity, which

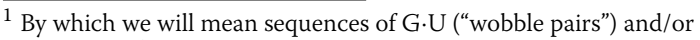
Watson-Crick pairs.
}

will be a molecule-by-molecule difference between the average ambiguity counts in and around native-structure stems and the average counts from elsewhere on the molecule. For now, we note that this measure, which we will call the ambiguity index and label $d$, depends on both the primary (" $p$ ") and native secondary (" $s$ ") structures of the molecule, which we emphasize by writing $d(p, s)$ rather than simply $d .^{2}$ To the extent that for any given native structure there is evolutionary pressure to minimize relative stem ambiguities, we expect to find small values of the ambiguity indexes.

But it would be a mistake to apply this line of thinking indiscriminately. The pathway to function for the many RNA molecules that operate as part of a larger, composite, complex of both RNA and protein componentsthe ribonucleoproteins, is considerably more complicated. The assembly of these complexes is far from fully worked out, but it stands to reason that the structures and folding of the component RNA molecules are influenced by the conformations of the accompanying proteins [8]. In such cases, the folding kinetics of the RNA molecule, as it might proceed in isolation and based only on thermodynamics and the free-energy landscape, may have little relevance to the in vivo assembly and arrival at a tertiary structure. Hence we will make a distinction between RNA molecules that are components of ribonucleoproteins (which we will refer to as "bound" RNA molecules) and RNA molecules which can function without being bound in a ribonucleoprotein complex (which we will refer to as "unbound" RNA molecules). The distinction is more relative than absolute. For example, many of the Group II introns both self-splice and reverse-splice, and both processes involve protein cofactors, some of which include a tight ribonculeoprotein complex with the maturase protein [7]. Nevertheless, we will treat these (as well as the Group I introns) as examples of "unbound," since most, if not all, can function without being bound to a specific protein [10], and since there is evidence that the adaptation of preexisting proteins to function in the splicing process evolved relatively recently [16].

The advantage of the two categories, bound and unbound, is that we can avoid making difficult absolute statements about the values of ambiguity indexes, per se, and instead focus on comparisons across the two populations. We reasoned that molecules from the bound (ribonculeoprotein) families would be less sensitive to the kinetic traps arising from ambiguities of their stem-producing subsequences than molecules from the unbound families. We therefore expected to find smaller ambiguity indexes in the unbound families. Recall now

\footnotetext{
${ }^{2}$ Native secondary structures often include so-called pseudoknots, which are sometimes excluded, or handled separately, for computational efficiency. Pseudoknots are formed from paired complementary subsequences and therefore included, by definition, in the ambiguity index.
} 
that the ambiguity index depends on both the primary and native secondary structures of the molecule, $d=d(p, s)$, which raises the question-which secondary structure $s$ should be used in the calculation? Our main conclusions were drawn using comparative secondary structures $[17$, 18] available through the RNA STRAND database[19], a curated collection of RNA secondary structures which are widely used as reference structures for single RNA molecules[20-22].

But this dependency on $s$ also afforded us the opportunity to make comparisons to a second, much-studied, approach to secondary structure prediction: equilibrium thermodynamics. The premise, namely that the structures of non-coding RNA molecules in vivo are in thermal equilibrium, is controversial. Nevertheless, variations on equilibrium methods constitute the prevailing computational approaches to predicting secondary structure. $^{3}$ Typically, these approaches use estimates of the conformation-dependent contributions to the free-energy and dynamic-programming type calculations to produce either samples from the resulting equilibrium distribution or minimum free energy (MFE) secondary structures $[23,24]$. Yet the biological relevance of equilibrium and minimum energy structures has been a source of misgivings at least since 1969, when Levinthal pointed out that the time required to equilibrate might be too long by many orders of magnitude [25]. In light of these observations, and considering the "frustrated" nature of the folding landscape, many have argued that when it comes to structure prediction for macromolecules, kinetic accessibility is more relevant than equilibrium thermodynamics [25-29]. In fact, a metastable state that is sufficiently long-lived and accessible might be biologically indistinguishable from an equilibrium state. Since the same issues of kinetic accessibility and the roles of kinetic traps that are behind these controversies are also behind our motivation to explore ambiguities, we also used the MFE secondary structure $s^{\prime}$, as estimated using standard packages, to compute a second ambiguity index for each RNA molecule: $d\left(p, s^{\prime}\right)$. In this way, we could look for differences, if any, between conclusions based on the comparative structure and those based on the MFE structure.

The choice of RNA families to represent the two groups was limited by the availability of reliable comparative secondary structures and the belief that the ambiguities captured by our index would be more relevant in large rather than small RNA molecules. With these considerations in mind, we chose the transfer-messenger RNAs (tmRNA), the RNAs of signal recognition particles (SRP RNA), the ribonuclease P family (RNase P), and the $16 \mathrm{~s}$

\footnotetext{
${ }^{3}$ Molecular dynamics, which might be called "agnostic" to the question of equilibrium, has proven to be exceedingly difficult, and has not yet yielded a useful tool for generic folding of large molecules.
}

and 23s ribosomal RNAs (16s and 23s rRNA) as representatives of "bound" (ribonucleoprotein) RNA molecules, and the Group I and Group II introns (sometimes referred to as self-splicing introns) as representatives of "unbound" molecules. See Methods for more details about the data set.

In summary, we will make a statistical investigation of the ambiguity index, as it varies between two groups of molecules (bound and unbound) and as it is defined according to either of two approaches to secondary structure prediction (comparative and MFE). In line with expectations, we will demonstrate that unbound molecules have systematically lower ambiguity indexes, when computed using comparative secondary structures, than bound molecules. The effect is strong: the average ambiguity in each unbound family is lower than the average ambiguity in every bound family. And the effect is still visible at the single-molecule level: a randomly chosen molecule can be accurately classified as belonging to the unbound group versus the bound group by simply thresholding on the ambiguity index (ROC area 0.81 ). We will also show that the utility of the ambiguity index to distinguish unbound from bound molecules disappears when the MFE structure is substituted for the comparative structure in computing the index. A related observation is that the ambiguity index of an unbound molecule can be used to classify whether the index itself was derived from the comparative versus MFE structure. To the extent that the comparative secondary structures are more accurate, these latter results might be interpreted as adding to existing concerns about the relevance of equilibrium RNA structures.

By using comparisons as opposed to absolute statistics, and various normalizations, and by favoring nonparametric (distribution-free) statistical methods, we have done our best to avoid subtle biases and hidden assumptions that would explain or at least influence the results. But more confidence would come with more data, especially more RNA families of both the ribonucleoprotein type and those that typically function without first forming tight assemblies with proteins. Given the rate of new discoveries and the rapid growth of accessible data sets, opportunities can not be far away.

The remainder of the paper is organized as follows: In the Results section we first develop some basic notation and definitions, and then present an exploratory and largely informal statistical analysis. This is followed by formal results comparing ambiguities in molecules drawn from the unbound families to those from the bound families, and then by a comparison of the ambiguities implied by secondary structures derived from comparative analyses to those derived through minimization of free energy. The Results section is followed by Discussion and Conclusions, in which we will recap the main results, further 
speculate about their interpretations, suggest refinements in the index that might highlight the effects of cotranscriptional folding and the varying thermodynamic stability of stems of different lengths, and review how our results bear on current thinking about RNA folding and structure. And finally, in Methods, we include detailed information about the data and its (open) source, as well as links to code that can be used to reproduce our results or for further experimentation.

\section{Results}

\section{Basic Notation and the Ambiguity Index}

Consider a non-coding RNA molecule with $N$ nucleotides. Counting from $5^{\prime}$ to $3^{\prime}$, we denote the primary structure by

$p=\left(p_{1}, p_{2}, \cdots, p_{N}\right)$, where $p_{i} \in\{A, G, C, U\}, i=1, \cdots, N$

and the secondary structure by

$s=\{(j, k):$ nucleotides $j$ and $k$ are paired, $1 \leq j<k \leq N\}$

Recall that we are interested in investigating the ambiguity of different subsequences in the RNA molecule. To formalize the notion of a subsequence, we define the segment at location $i$ to be

$$
P_{i}=\left(p_{i}, p_{i+1}, p_{i+2}, p_{i+3}\right) \text { for } i=1,2, \ldots, N-3
$$

In other words, the segment at location $i$ is the sequence of four consecutive nucleotides that starts at $i$ and proceeds from $5^{\prime}$ to $3^{\prime}$. There is no particular reason for using segments of length four, and in fact all qualitative conclusions are identical with segment lengths three, four, or five, and quite likely, many other larger lengths.

To study the ambiguity of a particular segment, we are interested in counting the locations which could feasibly form a stem with the given segment. We start by identifying which locations are viable to pair with $P_{i}$, based just on location and not nucleotide content. The only constraint on location is that an RNA molecule cannot form a loop of two or fewer nucleotides. Let $A_{i}$ be the set of all segments that are potential pairs of $P_{i}$ :

$$
\begin{gathered}
A_{i}=\left\{P_{j}: 1 \leq j \leq i-7(\text { segment precedes } i)\right. \text { or } \\
i+7 \leq j \leq N-3(\text { segment follows } i)\}
\end{gathered}
$$

We can now define the local ambiguity function,

$$
a(p)=\left(a_{1}(p), \cdots, a_{N-3}(p)\right)
$$

which is a vector-valued function of the primary structure $p$, and quantifies the ambiguities at different locations of the molecule. The vector has one component, $a_{i}(p)$, for each segment $P_{i}$, namely the number of feasible segments that are complementary to $P_{i}$ (allowing for G.U wobble pairings in addition to Watson-Crick pairings):

$$
\begin{aligned}
a_{i}(p)= & \#\left\{P \in A_{i}: P \text { and } P_{i} \text { are complementary }\right\} \\
= & \#\left\{P_{j} \in A_{i}:\left(p_{i, k}, p_{j, 5-k}\right) \in\{(A, U),(U, A),\right. \\
& (G, C),(C, G),(G, U),(U, G)\}, \\
& k=1, \ldots, 4\}
\end{aligned}
$$

Notice that $a_{i}(p)$ is independent of secondary structure $s$. It is simply the total number of subsequences that could form a stem structure with $\left(p_{i}, p_{i+1}, p_{i+2}, p_{i+3}\right)$.

We want to explore the relationship between ambiguity and secondary structure. We can do this conveniently, on a molecule-by-molecule basis, by introducing another vector-valued function, this time depending only on a purported secondary structure. Specifically, the new function assigns a descriptive label to each location (i.e. each nucleotide), determined by whether the segment at the given location is fully paired, partially paired, or fully unpaired.

Formally, given a secondary structure $s$, as defined in Eq (2), and a location $i \in\{1,2, \ldots, N-3\}$, let $f_{i}(s)$ be the number of nucleotides in $P_{i}$ that are paired under $s$ :

$f_{i}(s)=\#\left\{j \in P_{i}:(j, k) \in s\right.$ or $(k, j) \in s$, for some $\left.1 \leq k \leq N\right\}$

Evidently, $0 \leq f_{i}(s) \leq 4$. The "paired nucleotides function" is then the vector-valued function of secondary structure defined as $f(s)=\left(f_{1}(s), \ldots, f_{N-3}(s)\right)$. Finally, we use $f$ to distinguish three types of locations (and hence three types of segments): location $i$ will be labeled

$$
\left\{\begin{array}{c}
\text { single if } f_{i}(s)=0 \\
\text { double if } f_{i}(s)=4 \\
\text { transitional if } 0<f_{i}(s)<4
\end{array} \quad i=1,2, \cdots, N-3\right.
$$

In words, given a secondary structure, location $i$ is single if none of the four nucleotides $\left(p_{i}, p_{i+1}, p_{i+2}, p_{i+3}\right)$ are paired, double if all four are paired, and transitional if 1,2 , or 3 are paired.

\section{A First Look at the Data: Shuffling Nucleotides}

Our goals are to explore connections between ambiguities and basic characteristics of RNA families, as well as the changes in these relationships, if any, when using comparative as opposed to MFE secondary structures. For each molecule and each location $i$, the segment at $i$ has been assigned a "local ambiguity" $a_{i}(p)$ that depends only on the primary structure, and a label (single, double, or transitional) that depends only on the secondary structure. Since the local ambiguity, by itself, is strongly dependent on the length of the molecule, and possibly on other 
intrinsic properties, we define a relative ambiguity index: " $d_{T-S}(p, s)$ " which depends on both the primary $(p)$ and purported secondary $(s)$ structures:

$$
d_{\mathrm{T}-\mathrm{S}}(p, s)=\frac{\sum_{j=0}^{N-3} a_{j}(p) c_{j}^{\mathrm{tran}}(s)}{\sum_{j=0}^{N-3} c_{j}^{\text {tran }}(s)}-\frac{\sum_{j=0}^{N-3} a_{j}(p) c_{j}^{\text {single }}(s)}{\sum_{j=0}^{N-3} c_{j}^{\text {single }}(s)}
$$

where we have used $c_{i}^{\text {tran }}$ and $c_{i}^{\text {single }}$ for indicating whether location $i$ is transitional or single respectively. In other words, for each $i=1,2, \ldots, N-3$

$$
\begin{aligned}
c_{i}^{\operatorname{tran}}(s) & =\left\{\begin{array}{l}
1, \text { if location } i \text { is transitional } \\
0, \text { otherwise }
\end{array}\right. \\
c_{i}^{\text {single }}(s) & =\left\{\begin{array}{l}
1, \text { if location } i \text { is single } \\
0, \text { otherwise }
\end{array}\right.
\end{aligned}
$$

In short, the T-S ambiguity index is the difference in the averages of the local ambiguities at transitional sites and single sites.

We have also experimented with a second, closely related, index $d_{D-S}(p, s)$, in which averages over double locations replace averages over transitional locations. Since the definition is somewhat complicated by the observation that local ambiguities at double locations are almost always greater than one (the exceptions being certain configurations with bulges), and since the results using $d_{D-S}$ mirror those using $d_{T-S}$ (albeit somewhat weaker), we will focus exclusively on $d_{T-S}$. Results using $d_{D-S}$ can be accessed along with data and code, as explained in the Methods section. (Since there is only one index we could write $d$ in place of $d_{T-S}$, but chose to retain the subscript as a reminder of the source.)

Thinking kinetically, we might expect to find relatively small values of $d_{\mathrm{T}-\mathrm{S}}$, at least for molecules in the unbound families, as discussed in Background. One way to look at this is that larger numbers of partial matches for a given sequence in or around a stem would likely interfere with the nucleation of the native stem structure, and nucleation appears to be a critical and perhaps even rate-limiting step. Indeed, the experimental literature [30-33] has long suggested that stem formation in RNA molecules is a twostep process. When forming a stem, there is usually a slow nucleation step, resulting in a few consecutive base pairs at a nucleation point, followed by a fast zipping step. It is important to note, though, that the application of this line of reasoning to the $d_{T-S}(p, s)$ index requires that $s$ be an accurate representation of the native secondary structure. For the time being we will use the time-honored comparative structures for $s$, returning later to the questions about MFE structures raised in Background.

How are we to gauge $d_{\mathrm{T}-\mathrm{S}}$ and compare values across different RNA families? Consider the following experiment: for a given RNA molecule we create a "surrogate" which has the same nucleotides, and in fact the same counts of all four-tuple segments as the original molecule, but is otherwise ordered randomly. If ACCU appeared eight times in the original molecule, then it appears eight times in the surrogate, and the same can be said of all sequences of four successive nucleotides-the frequency of each of the $4^{4}$ possible segments is preserved in the surrogate. If we also preserve the locations of the transitional, double, and single labels (even though there is no actual secondary structure of the surrogate), then we can compute a new value for $d_{\mathrm{T}-\mathrm{S}}$, say $\tilde{d}_{\mathrm{T}-\mathrm{S}}$, from the surrogate. If we produce many surrogate sequences then we will get a sampling of $\tilde{d}_{\text {T-S }}$ values, one for each surrogate, to which we can compare $d_{\mathrm{T}-\mathrm{S}}$. We made several experiments of this type-one for each of the seven RNA families (Group I and Group II Introns, tmRNA, SRP RNA, RNase P, and 16s and 23s rRNA).

To make this precise, consider an RNA molecule with primary structure $p$ and comparative secondary structure $s$. Construct a segment "histogram function," $\mathcal{H}(p)$, which outputs the number of times that each of the $4^{4}$ possible segments appears in $p$. Let $\mathcal{P}(p)$ be the set of all permutations of the ordering of nucleotides in $p$, and let $\mathcal{E}(p) \subseteq \mathcal{P}(p)$ be the subset of permutations that preserve the frequencies of four-tuples. If, for example, $p=(A, A, U, A, A, U, U, A, A)$, then there are six fourtuples, $(A, A, U, A),(A, U, A, A),(U, A, A, U),(A, A, U, U)$, ( $A, U, U, A),(U, U, A, A)$, and each happens to appear only once, i.e., the histogram function $\mathcal{H}(p)$ assigns the number one to each of these six four-tuples and zero to every other four-tuple. The only additional sequence that preserves these frequencies (aside from $p$ itself) turns out to be $p^{\prime}=(A, A, U, U, A, A, U, A, A)$, and in this example $\mathcal{E}(p)=\left\{p, p^{\prime}\right\}$. More generally

$$
\mathcal{E}(p)=\left\{p^{\prime} \in \mathcal{P}(p): \mathcal{H}\left(p^{\prime}\right)=\mathcal{H}(p)\right\}
$$

Clever algorithms (all of which are variants and generalizations of the Euler algorithm, e.g. see [36] and references therein) exist for efficiently drawing independent samples from the uniform distribution on $\mathcal{E}$-see [34-36]. Let $p^{(1)}, \ldots, p^{(K)}$ be $K$ such samples, and let $d_{\mathrm{T}-\mathrm{S}}\left(p^{(1)}, s\right), \ldots, d_{\mathrm{T}-\mathrm{S}}\left(p^{(K)}, s\right)$ be the corresponding T-S ambiguity indexes. Whereas the secondary structure $s$ remains the same across shuffles, the local ambiguity function $a\left(p^{(k)}\right)$, which depends on the primary structure, changes with $k$, and so does the resulting ambiguity index $d_{\mathrm{T}-\mathrm{S}}\left(p^{(k)}, s\right)$. How different is $d_{\mathrm{T}-\mathrm{S}}(p, s)$ from the ensemble of values $d_{\mathrm{T} \text {-S }}\left(p^{(k)}, s\right)$ derived by sampling from $\mathcal{E}(p)$ ? To measure this, let $\alpha_{\mathrm{T}-\mathrm{S}}(p, s) \in[0,1]$ be the lefttail empirical probability of choosing an ambiguity index less than or equal to $d_{\mathrm{T}-\mathrm{S}}(p, s)$ from the ensemble of values 


$$
\begin{aligned}
& \left\{d_{\mathrm{T}-\mathrm{S}}(p, s), d_{\mathrm{T}-\mathrm{S}}\left(p^{(1)}, s\right), \ldots, d_{\mathrm{T}-\mathrm{S}}\left(p^{(K)}, s\right)\right\}: \\
& \alpha_{\mathrm{T}-\mathrm{S}}(p, s)=\frac{1+\#\left\{k \in\{1, \ldots, K\}: d_{\mathrm{T}-\mathrm{S}}\left(p^{(k)}, s\right) \leq d_{\mathrm{T}-\mathrm{S}}(p, s)\right\}}{1+K}
\end{aligned}
$$

In essence, for each RNA family the $\alpha$ score is a selfcalibrated ambiguity index. The results are not very sensitive to $K$ nor to the particular sample, provided that $K$ is large enough. We used $K=10,000$.

If the number of distinct sequences in $\mathcal{E}(p)$ is small, then so is the number of possible values of $\alpha$. In such cases, $\alpha$ will be of little value for comparing ambiguity indexes across types of molecules or proposed secondary structures. Indeed, many short sequences, such as $p=$ ( $A, C, G, U, A, C, G, U$ ), have no histogram-preserving primary structures beyond $p$ itself. But as we have already remarked, our methods are motivated by a kinetic viewpoint, within which the greatest challenges to folding are faced by the larger rather than smaller molecules. Hence, our experiments are with sequences that are relatively long. In fact, none of the RNA families used in our experiments have a median length shorter than 274 nucleotides, and most are much longer-see Table 4 . At these lengths it is extremely rare that a sample of 10,000 primary sequences from $\mathcal{E}(p)$ will have any duplicates. Hence there is no built-in meaningful loss of resolution in the $\alpha$ statistic.

It is tempting to interpret $\alpha_{\mathrm{T}-\mathrm{S}}(p, s)$ as a $\mathrm{p}$-value from a conditional hypothesis test: Given $s$ and $\mathcal{H}$, test the null hypothesis that $d_{\mathrm{T}-\mathrm{S}}(p, s)$ is statistically indistinguishable from $d_{\mathrm{T}-\mathrm{S}}\left(p^{\prime}, s\right)$, where $p^{\prime}$ is a random sample from $\mathcal{E}$. If the alternative hypothesis were that $d_{\mathrm{T}-\mathrm{S}}(p, s)$ is too small to be consistent with the null, then the null is rejected in favor of the alternative with probability $\alpha_{\mathrm{T}-\mathrm{S}}(p, s)$. The problem with this interpretation is that this null hypothesis violates the observation that given $\mathcal{H}$ there is information in $s$ about $p$, whereas $p^{(1)}, \ldots, p^{(K)}$ are independent of $s$ given $\mathcal{H}$. In other words, $d_{\mathrm{T}-\mathrm{S}}(p, s)$ and $d_{\mathrm{T}-\mathrm{S}}\left(p^{\prime}, s\right)$ have different conditional distributions given $s$ and $\mathcal{H}$, in direct contradiction to the null hypothesis. A larger problem is that there is no reason to believe the alternative; we are more interested in relative than absolute ambiguity indexes. Thinking of $\alpha_{\mathrm{T}-\mathrm{S}}(p, s)$ as a calibrated intra-molecular index, we want to know how $\alpha_{\mathrm{T}-\mathrm{s}}(p, s)$ varies across RNA families, and whether these variations depend on the differences between comparative and MFE structures.

Nevertheless, $\alpha_{\mathrm{T}-\mathrm{S}}(p, s)$ is a useful statistic for exploratory analysis. Table 1 provides summary data about the $\alpha$ scores for each of the seven RNA families. For each molecule in each family we use the primary structure and the comparative secondary structure, and $K=10,000$ samples from $\mathcal{E}$, to compute individual T-S
Table 1 Comparative Secondary Structures: calibrated ambiguity indexes, by RNA family

\begin{tabular}{llll}
\hline Family & $\begin{array}{l}\text { Number } \\
\text { molecules }\end{array}$ & $\begin{array}{l}\text { Median } \\
\text { length }\end{array}$ & $\begin{array}{l}\text { Median } \\
\alpha_{\text {T-S }}\end{array}$ \\
\hline Group I Introns & 116 & 451 & 0.432 \\
Group II Introns & 34 & 990 & 0.181 \\
tmRNA & 404 & 363 & 0.926 \\
SRP RNA & 346 & 274 & 0.790 \\
RNase P & 407 & 330 & 0.925 \\
16s rRNA & 279 & 1512 & 0.938 \\
23s rRNA & 59 & 2913 & 1.000 \\
\hline
\end{tabular}

The number of molecules, the median length (number of nucleotides), and the median $\alpha$ scores for the T-S ambiguity indexes (Eq. 11) for each of the seven RNA families studied. RNA molecules from the first two families (unbound) are active without necessarily forming ribonucleoprotein complexes; the remaining five are bound in ribonucleoproteins. Molecules from the unbound families have lower ambiguity indexes

scores (Eq 11). Keeping in mind that a smaller value of $\alpha$ represents a smaller calibrated value of the corresponding ambiguity index $d(p, s)$, there is evidently a disparity between ambiguity indexes of RNA molecules that form ribonucleoproteins and those that are already active without forming a ribonculeoprotein complex. As a group, unbound molecules have systematically lower ambiguity indexes. As already noted, this observation is consistent with, and in fact anticipated by, a kinetic point of view. Shortly, we will further support this observation with ROC curves and rigorous hypothesis tests.

Does the MFE structure similarly separate single-entity RNA molecules from those that form ribonucleoproteins? A convenient way to explore this question is to recalculate and recalibrate the ambiguity indexes of each molecule in each of the seven families, but using the MFE in place of the comparative secondary structures. The results are summarized in Table 2. By comparison to the results

Table 2 MFE Secondary Structures: calibrated ambiguity indexes, by RNA family

\begin{tabular}{llll}
\hline Family & $\begin{array}{l}\text { Number } \\
\text { molecules }\end{array}$ & $\begin{array}{l}\text { Median } \\
\text { length }\end{array}$ & $\begin{array}{l}\text { Median } \\
\alpha \text { T-S }\end{array}$ \\
\hline Group I Introns & 116 & 451 & 0.833 \\
Group II Introns & 34 & 990 & 0.841 \\
tmRNA & 404 & 363 & 0.867 \\
SRP RNA & 346 & 274 & 0.803 \\
RNase P & 407 & 330 & 0.955 \\
16s rRNA & 279 & 1512 & 0.982 \\
23s rRNA & 59 & 2913 & 1.000 \\
\hline
\end{tabular}

Identical to Table 1, except that the ambiguity indexes and their calibrations are calculated using the MFE secondary structures rather than comparative analyses. There is little evidence in the MFE secondary structures for lower ambiguity indexes among the unbound RNA molecules 
shown from Table 1, the separation of unbound from bound molecules nearly disappears when viewed under the MFE secondary structures. Possibly, the comparative structures, as opposed to the MFE structures, better anticipate the need to avoid kinetic traps in the folding landscape. Here too we will soon revisit the data using ROC curves and proper hypothesis tests.

\section{Formal Statistical Analyses}

The T-S ambiguity index $d_{\mathrm{T}-\mathrm{S}}(p, s)$ is an intra-molecular measure of the difference between the number of available double-stranded Watson-Crick and wobble pairings for segments in and around stems and pseudoknots versus segments within single-stranded regions. As such, $d_{\mathrm{T} \text {-S }}$ depends on both $p$ and any purported secondary structure, $s$. Based on a calibrated version, $\alpha_{\mathrm{T}-\mathrm{s}}(p, s)$, and employing the comparative secondary structure for $s$, we found support for the idea that non-coding RNA molecules in the unbound families, which are active absent participation in ribonucleoproteins, are more likely to have small ambiguity indexes than RNA molecules that operate exclusively as part of ribonucleoproteins. Furthermore, the difference appears to be sensitive to the approach used for identifying secondary structure-there is little, if any, evidence in indexes $d_{\text {T-S }}$ derived from the MFE secondary structures for lower ambiguities among unbound molecules.

These qualitative observations can be used to formulate precise statistical hypothesis tests. Many tests come to mind, but perhaps the simplest and most transparent are based on nothing more than the molecule-by-molecule signs of the ambiguity indexes. Whereas ignoring the actual values of the indexes is inefficient in terms of information, and probably also in the strict statistical sense, tests based on signs require very few assumptions and are, therefore, more robust to model misspecification. All of the p-values that we will report are based on the hypergeometric distribution, which arises as follows.

We are given a population of $M$ molecules, $m=$ $1, \ldots, M$, each with a binary outcome measure $B_{m} \in$ $\{-1,+1\}$. There are two subpopulations of interest: the first $M_{1}$ molecules make up population 1 and the next $M_{2}$ molecules make up population 2; $M_{1}+M_{2}=M$. We observe $n_{1}$ plus values in population 1 and $n_{2}$ in population 2

$$
\begin{aligned}
& n_{1}=\#\left\{m \in\left\{1,2, \ldots, M_{1}\right\}: B_{m}=+1\right\} \\
& n_{2}=\#\left\{m \in\left\{M_{1}+1, M_{1}+2, \ldots, M\right\}: B_{m}=+1\right\}
\end{aligned}
$$

We suspect that population 1 has less than its share of plus ones, meaning that the $n_{1}+n_{2}$ population of plus ones was not randomly distributed among the $M$ molecules. To be precise, let $N$ be the number of plus ones that appear from a draw, without replacement, of $M_{1}$ samples from
$B_{1}, \ldots, B_{M}$. Under the null hypothesis, $H_{o}, n_{1}$ is a sample from the hypergeometric distribution on $N$ :

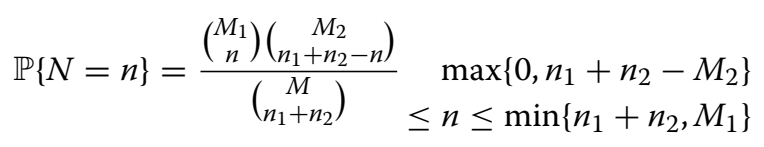

The alternative hypothesis, $H_{a}$, is that $n_{1}$ is too small to be consistent with $H_{o}$, leading to a left-tail test with p-value $\mathbb{P}\left\{N \leq n_{1}\right\}$ (which can be computed directly or using a statistical package, e.g. hypergeom.cdf in scipy.stats).

It is by now well recognized that $\mathrm{p}$-values should never be the end of the story. One reason is that any departure from the null hypothesis in the direction of the alternative, no matter how small, is doomed to be statistically significant, with arbitrarily small p-value, once the sample size is sufficiently large. In other words, the effect size remains hidden. Therefore, in addition to reporting $\mathrm{p}$-values, we will also display estimated ROC curves, summarizing performance of two related classification problems: (i) Classify a single RNA molecule, randomly selected from the seven families, as belonging to the unbound group or the bound group based only on thresholding $d_{\mathrm{T}-\mathrm{S}}(p, s)$. Compare performance under each of the two secondarystructure models, comparative and MFE; and (ii) Randomly select an RNA molecule from the unbound group and classify the origin of its secondary structure (comparative or MFE), here again based only on thresholding $d_{\mathrm{T} \text {-S }}(p, s)$. Now Repeat the process, but selecting randomly from the bound group.

\section{Bound versus Unbound}

Classification. Consider an RNA molecule, $m$, selected from one of the seven families in our data set, with primary structure $p$ and secondary structure $s$ computed by comparative analysis. Given only the T-S ambiguity index of $m$ (i.e. given only $d_{\mathrm{T}-\mathrm{S}}(p, s)$ ), how accurately could we classify the origin of $m$ as the unbound versus bound group? The foregoing exploratory analysis suggests constructing a classifier that declares a molecule to be unbound when $d_{\mathrm{T}-\mathrm{S}}(p, s)$ is small, e.g. $d_{\mathrm{T}-\mathrm{S}}(p, s)<$ $t$, where the threshold $t$ governs the familiar trade off between rates of "true positives" (an unbound molecule $m$ is declared 'unbound') and "false positives" (a bound molecule $m$ is declared 'unbound'). Small values of $t$ favor low rates of false positives at the price of low rates of true positives, whereas large values of $t$ favor high rates of true-positives at the price of high rates of false positives. Since for each molecule $m$ we have both the correct classification (unbound or bound) and the statistic $d$, we can estimate the ROC performance of our threshold classifier by plotting the empirical values of the pair

(\# false positives, \# true positives) 
for each value of $t$. The ROC curve for the two-category (unbound versus bound) classifier based on thresholding $d_{\mathrm{T}-\mathrm{S}}(p, s)<t$ is shown in the left panel of Fig. 1. Also shown is the estimated area under the curve (AUC=0.81), which has a convenient and intuitive interpretation, as it is equal to the probability that for two randomly selected molecules, $m$ from the unbound population and $m^{\prime}$ from the bound population, the T-S ambiguity index of $m$ will be smaller than the T-S ambiguity index of $m^{\prime}$.

$\boldsymbol{p}$-Values. As mentioned earlier, we can also associate a traditional $\mathrm{p}$-value to the problem of separating unbound from bound molecules, based again on the T$\mathrm{S}$ ambiguity indexes. We consider only the signs (positive or negative) of these indexes, and then test whether there are fewer than expected positive indexes among the unbound as opposed to the bound populations. This amounts to computing $\mathbb{P}\left\{N \leq n_{1}\right\}$ from the hypergeometric distribution-Eq (14). The relevant statistics can be found in Table 3, under the column labels \#mol's and $\# \boldsymbol{d}_{\text {T-S }}>\mathbf{0}$. Specifically, $M_{1}=116+34=150$ (number of unbound molecules), $M_{2}=404+346+$ $407+279+59=1495$ (number of bound molecules), $n_{1}=50+8=58$ (number of positive T-S indexes among unbound molecules) and $n_{2}=368+269+$ $379+210+53=1279$ (positive bound indexes). The resulting $\mathrm{p}$-value, $1.2 \cdot 10^{-34}$, is essentially zero, meaning that the positive T-S indexes are not distributed proportional to the sizes of the unbound and bound populations, which is by now obvious in any case. To repeat our caution, small p-values conflate sample size with effect size, and for that reason we have chosen additional ways, using permutations as well as classifications, to look at the data.

\section{Comparative versus Minimum Free Energy}

As we have just seen, ambiguity indexes based on MFE secondary structures, as opposed to comparative secondary structures, do not make the same stark distinction between unbound and bound RNA molecules. To explore this a little further, we can turn the analyses of the previous paragraphs around and ask to what extent knowledge of the ambiguity index is sufficient to predict the source of a secondary structure-comparative or free energy? This turns out to depend on the group from which the molecule was drawn: The ambiguity index is strongly predictive among unbound molecules and, at best, weakly predictive among bound molecules.

Consider the two ROC curves in Fig. 2. In each of the two experiments a classifier was constructed by thresholding the T-S ambiguity index, declaring the secondary structure, $s$, to be "comparative" when $d_{\mathrm{T}-\mathrm{S}}(p, s)<t$ and "MFE" otherwise. The difference between the two panels is in the population used for the classification experiments-unbound molecules in the left-hand panel (AUC $=0.81$ ) and bound molecules in the right-hand panel (AUC $=0.54$, barely above chance). The corresponding hypothesis tests seek evidence against the null hypotheses that in a given group (unbound or bound) the set of positive T-S ambiguity indexes $\left(d_{\mathrm{T}-\mathrm{S}}(p, s)>0\right)$ are equally distributed between the comparative and freeenergy derived indexes, and in favor of the alternatives that the T-S ambiguity indexes are less typically positive
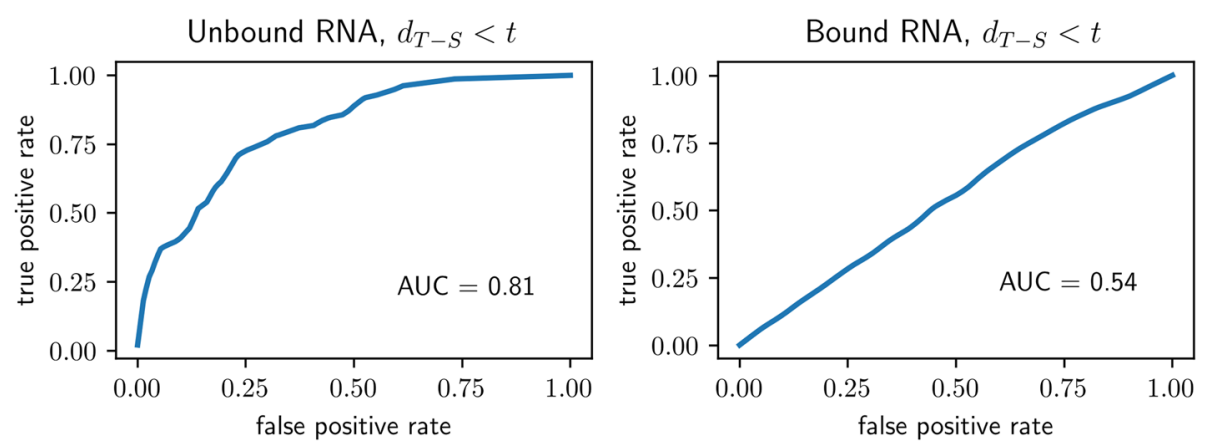

Fig. 1 Unbound or Bound? ROC performance of classifiers based on thresholding the T-S ambiguity index. Small values of $d_{T-S}(p, s)$ are taken as evidence that a molecule belongs to the unbound group as opposed to the bound group. In the left panel, the classifier is based on using the comparative secondary structure for s to compute the ambiguity index. Alternatively, the MFE structure is used for the classifier depicted in the right panel. AUC: Area Under Curve - see text for interpretation. Additionally, for each of the two experiments, a p-value was calculated based only on the signs of the individual ambiguity indexes, under the null hypothesis that positive indexes are distributed randomly among molecules in all seven RNA families. Under the alternative, positive indexes are more typically found among the unbound as opposed to bound families. Under the null hypothesis the test statistic is hypergeometric - see Eq 14. Left Panel: $p=1.2 \times 10^{-34}$. Right Panel: $p=0.02$. In considering these $p$-values, it is worth re-emphasizing the points made about the interpretation of $p$-values in the paragraph following Eq 14. The right panel illustrates the point: the ambiguity index based on the MFE secondary structure "significantly distinguishes the two categories $(p=0.02)$ " but clearly has no utility for classification. (These ROC curves and those in Fig. 2 were lightly smoothed by the method known as "Locally Weighted Scatterplot Smoothing," e.g. with the python command $Y=\operatorname{lowess}(Y, X, 0.1$, return_sorted=False) coming from statsmodels.nonparametric.smoothers_lowess) 
Table 3 Numbers of Positive Ambiguity Indexes, by family

\begin{tabular}{llll}
\hline Family & $\#$ mol's & $\# d_{\text {T-S }}>0$ & $\# d_{\text {T- }}>0$ \\
\hline Group I Introns & 116 & 50 & 94 \\
Group II Introns & 34 & 8 & 27 \\
tmRNA & 404 & 368 & 358 \\
SRP RNA & 346 & 269 & 264 \\
RNase P & 407 & 379 & 377 \\
16s rRNA & 279 & 210 & 254 \\
23s rRNA & 59 & 53 & 54 \\
\hline
\end{tabular}

\#mol's: number of molecules; \#dT-S $>0$ : numbers of positive T-S ambiguity indexes, secondary structures computed by comparative analysis; $\# d_{\hat{T}_{-}-\bar{S}}>0$ : numbers of positive T-S ambiguity indexes, secondary structures computed by minimum free energy

for the comparative secondary structures. The necessary data can be found in Table 3 . The test results are consistent with the classification experiments: the hypergeometric pvalue is is $5.4 \cdot 10^{-14}$ for the unbound population and 0.07 for the bound population.

Qualitatively, these various ROC and p-value results were easy to anticipate from even a superficial examination of Table 3. Start with the first two rows (unbound molecules): A relatively small fraction of unbound molecules have positive ambiguities when the index is computed from comparative analyses, whereas most of these same molecules have positive ambiguities when the index is computed from MFE structures. Looking across the next five rows (bound molecules), no such trend is discernible. Similarly, from a glance at the column labeled $\# d_{\text {T-S }}>0$ (derived from comparative analyses) it is apparent that the fraction of positive indexes among the unbound molecules is much lower than among the bound molecules. What's more, this effect is missing in the MFE indexes (column labeled $\# d_{\tilde{\mathrm{T}}-\tilde{\mathrm{S}}}>0$ ). ${ }^{4}$

\section{Discussion}

Consider a non-coding RNA molecule with a native tertiary structure that is active, in vivo, without necessarily being tightly bound with other molecules in a ribonucleoprotein complex. We have labeled these molecules "unbound" and reasoned that there are likely relationships between their primary and secondary structures that not only support the tertiary structure, but also the folding process by which it emerges. Specifically, we reasoned that examination of the primary and native secondary structures might reveal evolutionary mechanisms that discourage disruptive kinetic traps. Conjecturing that the availability of non-native pairings for subsequences that are part of the native secondary structure would be particularly disruptive, we defined an intra-molecular index

\footnotetext{
${ }^{4}$ The specific values of the areas under the ROC curves depend on the specific values of the indexes. The equality-to two digits-of the areas in the left-hand panels of Figs. 2 and 1 is a coincidence.
}

that we called the ambiguity index. The ambiguity index is a function of a molecule's primary and native secondary structures devised so that lower values of the index reflect fewer opportunities for stem participating subsequences to pair elsewhere in the molecule. We examined the Group I and Group II introns, two families of molecules that are believed to perform some of their functions (namely self splicing) in an "unbound" state, to see if their ambiguity indexes were lower than might be expected were there no such evolutionary pressures to protect stem structures. Heuristic permutation-type tests appeared to confirm our expectation that these molecules would have low ambiguities.

We sought additional evidence in two directions. The first was to compare ambiguity indexes in unbound molecules to those in "bound" molecules, i.e. molecules that are known to function as part of ribonucleoprotein complexes where the argument against these particular kinds of ambiguities is weaker. We found a strong separation between the unbound and bound molecules, the former having substantially lower indexes. This was demonstrated by statistical tests and, perhaps more meaningfully, by showing that the ambiguity index could be used to classify with good accuracy individual molecules as either bound or unbound. These experiments were based on comparative secondary structures available through the RNA STRAND database[19], which remains one of the most trusted sources for RNA secondary structures of single molecules[20-22].

In a second approach to additional evidence we substituted the comparative secondary structures with ones that were derived from approximations to the thermodynamic equilibrium structure (minimum free energy"MFE" structures). Though less accurate, MFE and related equilibrium-type structures are easy and quick to compute. But one line of thinking is that active biological structures are determined more by kinetic accessibility than thermodynamic equilibrium per se[25-29]. Biological stability is relative to biological timescale; the folding of any particular RNA could just as well end in metastability, provided that the process is repeatable and the result sufficiently stable over the molecule's proper biological lifetime. Indeed, it would be arguably easier to evolve an effective tertiary structure without the additional and unnecessary burden of thermal equilibrium. To the extent that kinetic accessibility and metastability might be more relevant than thermodynamic equilibrium, there would be little reason to expect the ambiguity index to make the same separation between unbound and bound molecules when derived from MFE structures instead of comparative structures. The results were consistent with this point of view-ambiguity indexes based on MFE structures make weak classifiers. We were surprised by the strength of the effect. After all, MFE structures are superficially quite 

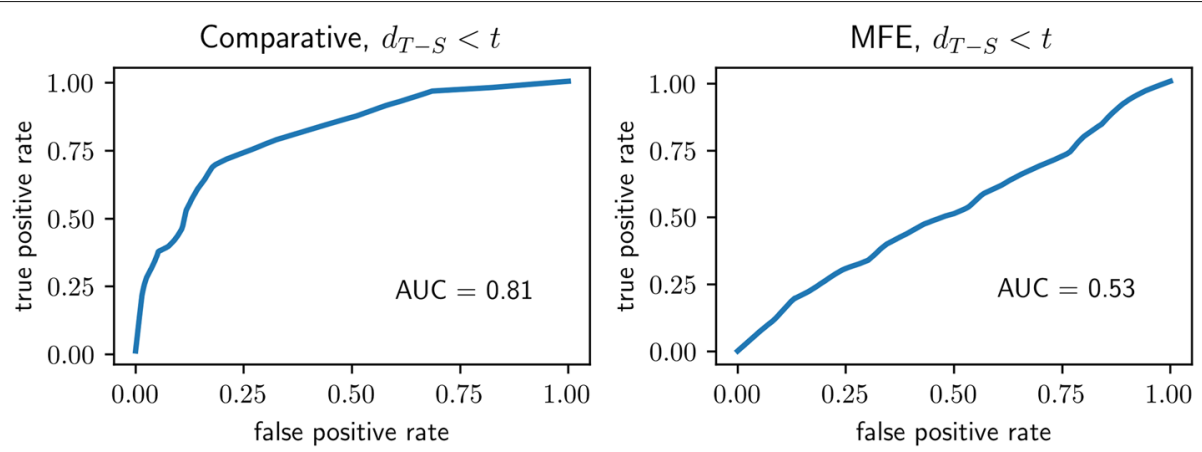

Fig. 2 Comparative or MFE? As in Fig. 1, each panel depicts the ROC performance of a classifier based on thresholding the T-S ambiguity index, with small values of $d_{T-S}(p, s)$ taken as evidence that $s$ was derived by comparative as opposed to MFE secondary structure analysis. Left Panel: performance on molecules chosen from the unbound group. Right Panel: performance on molecules chosen from the bound group. Conditional p-values were also calculated, using the hypergeometric distribution and based only on the signs of the indexes. In each case the null hypothesis is that comparative secondary structures are as likely to lead to positive ambiguity indexes as are MFE structures, whereas the alternative is that positive ambiguity indexes are more typical when derived from MFE structures. Left Panel: $p=5.4 \times 10^{-14}$. Right Panel: $p=0.07$

similar to comparative structures, yet the classification performance goes from strong (> 80\% AUC) to negligible (53\% AUC, just above chance). A worthwhile follow-up would be to examine the actual differences in secondary structure (as was done, with similar motivation but different tools, in [29]) in an effort to discern how they impact ambiguity.

A possible source of bias that might partially explain the strength of the observed effects was raised by an anonymous reviewer, who noted that the RNAfold program in the ViennaRNA package [20], used here to compute MFE structures, does not allow pseudoknots, a structural feature that is commonly present in comparative structures. To explore the possible effect of pseudoknots on our results, and to make for something closer to an "applesto-apples" comparison, we re-ran the experiments after removing all pseudoknots from the comparative structures $^{5}$. There were only small changes in the results-e.g. classification performance, "Bound or Unbound" (Fig. 1) using comparative structures went from $81 \%$ AUC to $79 \%$ AUC, whereas performance using MFE stayed the same at $53 \% \mathrm{AUC}^{6}$. Of course it is still possible that a true MFE structure, computed without compromises in the structure of the energy and allowing for pseudoknots, were it computable, would fare better in these experiments.

Another interesting point raised by the same reviewer concerns the well-known heterogeneity of structures within the Group I and Group II Introns, which constitute our unbound samples. In particular, these groups can be further divided into subgroups that have very different secondary structures (see Table 2 of [43]). To what

\footnotetext{
${ }^{5}$ Using methods presented in [37].

${ }^{6}$ More comprehensive results for the experiments with pseudoknot-free comparative secondary structures and detailed results for thirteen different unbound subgroups of RNA molecules can be accessed along with data and code-see Methods.
}

extent are the differences between bound and unbound molecules consistent across subgroups? To investigate this we re-computed the $\alpha_{\text {T-S }}$ indexes reported in Table 1, but this time for each subgroup of each of the Group I and Group II introns. The stark differences between bound and unbound molecules remain. In fact, the differences are more extreme for all but two of the unbound subgroups (Group IC1 and Group IIA), out of the thirteen available in our dataset ${ }^{6}$.

It has often been argued (e.g. [38, 39]) that the MFE structure itself may be a poor representative of thermal equilibrium. It is possible, then, that our observations to the effect that comparative and MFE structures have substantially different relationships to the ambiguity indexes, and our interpretation that comparative structures better separate unbound from bound molecules, would not hold up as well if we were to adopt a more ensembleoriented structure in place of the MFE, as advocated by [40], for example. In a related vein, and also within the context of thermodynamic equilibrium, Lin et al. [41] have given evidence that competing stems which are inconsistent may both contain a high measure of information about the equilibrium distribution, suggesting that in such cases both forms could be active and the notion of single (locations we have labeled "S") might itself be ambiguous. Certainly there are RNA molecules (e.g. riboswitches) that are active in more than one structural conformation. For such molecules, ambiguity is essential for their biological functioning, yet one would need to rethink the definition of an ambiguity index.

The ambiguity index $d_{\mathrm{T}-\mathrm{S}}$ is derived from the difference in average ambiguities of subsequences partly paired in the native structure (" $T$ ", transition locations) from those not paired in the native structure (single locations). We expected these differences to be small in unbound as opposed to bound molecules because we expected the 
stem structures to be more protected from non-native pairings. But this coin has another side: low ambiguities at unpaired (single) locations of bound molecules relative to unbound molecules would have the same effect. As an example, some unpaired RNA sequences may be critical to function, as in the messenger RNA-like region ("MLR") of tmRNA, and therefore relatively unambiguous. Also, it is possible that the formation of non-native stems among single-type subsequences are particularly disruptive to, perhaps even stereochemically preventing, the binding of an RNA molecule into a ribonucleoprotein complex. More generally, it is reasonable to assume that different evolutionary forces are at play for molecules destined to operate as parts of ribonucleoprotein complexes. In any case, the folding story may be even more complicated, or at least quite different, for the ribonculeoprotein RNAs.

Finally, we note that the ambiguity index, as currently formulated, is symmetric in the sense that there is no explicit difference in contributions from different locations along the $5^{\prime}$ to $3^{\prime}$ axis. Yet cotranscriptional folding, which appears to be nearly universal in non-coding RNA [42] strongly suggests that not all ambiguities are equally disruptive. Indeed, some non-native pairings between two subsequences, one of which is near the $3^{\prime}$ end of the molecule, might have been rendered stereochemically impossible before the $3^{\prime}$ half has even been transcribed. In addition, the current ambiguity index is calculated using segments of a fixed length (four for the results presented in the paper). Yet thermodynamic stability increases with stem lengths, which suggests that non-native pairings between two longer subsequences would be more disruptive than those between shorter subsequences. Possibly, a proper weighting of ambiguities coming from segments of different lengths would bring new insights. These further considerations open many new lines of reasoning, most of which suggest alternative indexes that could be statistically explored, especially as the data bank of known structures and functions continues to grow.

Overall, our results are consistent in supporting a role for kinetic accessibility that is already visible in the relationship between primary and secondary structures. Stronger evidence will require more bound and unbound families. The limiting factors, as of today, are the availability of families with large RNA molecules for which the comparative structures have been worked out and largely agreed upon.

\section{Conclusions}

In this paper, we have presented a statistical analysis of the relationship between the primary and secondary structures of non-coding RNA molecules. The results suggest that stem-disrupting kinetic traps are substantially less prevalent in molecules not participating in RNP complexes. In that this distinction is apparent under the comparative but not the MFE secondary structure, the results highlight a possible deficiency in structure predictions when based upon assumptions of thermodynamic equilibrium.

\section{Methods \\ Datasets}

We obtained comparative-analysis secondary structure data for seven different families of RNA molecules from the RNA STRAND database[19], a curated collection of RNA secondary structures which are widely used as reference structures for single RNA molecules[20-22]. These families include: Group I Introns and Group II Introns[43], tmRNAs and SRP RNAs[44], the Ribonuclease P RNAs[45], and 16s rRNAs and 23s rRNAs[43]. Table 4 contains information about the numbers and lengths (measured in nucleotides) of the RNA molecules in each of the seven families. Note that we excluded families like tRNAs, $5 \mathrm{~s}$ rRNAs and hammerhead ribozymes since most of the molecules in these families are too short to be of interest for our purpose. Also, since we are focusing on comparative-analysis secondary structures, to be consistent, we excluded any secondary structures derived from X-ray crystallography or NMR structures.

Note that Group I and Group II Introns are the only available families of unbound RNAs suitable for our analysis. There are some other families of unbound RNAs (e.g. ribozymes), but most of these RNAs are too short in length, and many of the structures are not derived using comparative analysis. Hence they are not included.

\section{RNA Secondary Structure Prediction Methods}

Comparative analysis[46] is based on the simple principle that a single RNA secondary structure can be formed from different RNA sequences. Using alignments of homologous sequences, comparative analysis has proven to be highly accurate in determining RNA secondary structures [18]. We used a large set of RNA secondary structures

Table 4 Data Summary

\begin{tabular}{lllll}
\hline Family & Number & Min length & Max length & Median \\
\hline Group I Introns & 116 & 210 & 2630 & 451 \\
Group II Introns & 34 & 619 & 2729 & 990 \\
tmRNA & 404 & 102 & 437 & 363 \\
SRP RNA & 346 & 66 & 533 & 274 \\
RNase P & 407 & 189 & 486 & 330 \\
16s rRNA & 279 & 612 & 2394 & 1512 \\
23s rRNA & 59 & 953 & 4381 & 2913
\end{tabular}

The seven families of RNA used in the experiments. Table includes the number of molecules in each family, as well as basic statistics about the numbers of nucleotides in the primary sequence of each of the molecules. Data was downloaded from the http://www.rnasoft.ca/strand/ 
determined by comparative analyses to serve as ground truth.

When it comes to computational prediction of RNA secondary structures, exact dynamic programming algorithms based on carefully measured thermodynamic parameters make up the most prevalent methods. There exist a large number of software packages for the energy minimization [20, 38, 47-51]. In this paper, we used the ViennaRNA package [20] to obtain the MFE secondary structures for our statistical analysis.

\section{Reproducing the Results}

The results presented in this paper, as well as additional results on experiments with the D-S ambiguity index, pseudoknot-free comparative secondary structures, and detailed results for thirteen different unbound subgroups of RNA molecules, can be easily reproduced. Follow the instructions on https://github.com/StannisZhou/rna statistics. Here we make a few comments regarding some implementation details.

- In the process of obtaining the data, we used the bpseq format, and excluded structures derived from $\mathrm{X}$-ray crystallography or NMR structures, as well as structures for duplicate sequences. Concretely, this means picking a particular type, and select No for Validated by NMR or X-Ray and Non-redundant sequences only for Duplicates on the search page of the RNA STRAND database. A copy of the data we used is included in the GitHub repository, but the same analyses can be easily applied to other data.

- When processing the data, we ignored molecules for which we have nucleotides other than $A, G, C, U$, and molecules for which we don't have any base pairs.

- When comparing the local ambiguities in different regions of the RNA molecules, we ignored molecules for which we have empty regions (i.e. at least one of single, double and transitional is empty), as well as molecules where all local ambiguities in single or double regions are 0 .

- For shuffling primary structures, we used an efficient and flexible implementation of the Euler algorithm[34-36] called uShuffle [52], which is conveniently available as a python package.

- For removing pseudoknots from comparative secondary structures, we used the standalone implementation of methods proposed in [37]. The actual pseudoknot-free comparative secondary structures used in our experiments are available at https://github.com/StannisZhou/rna_statistics/tree/ master/data_without_pseudoknots.

\section{Abbreviations}

AUC: Area under the curve; MFE: Minimum free energy; MLR: Messenger RNA-like region; RNase P: Ribonuclease P; RNP: Ribonucleoprotein; ROC:
Receiver operating characteristic; rRNA: Ribosomal RNA; SRP: Signal recognition particles; tmRNA: Transfer-messenger RNA

\section{Acknowledgements}

The authors would like to thank Yang Chen for helpful discussions and Matthew T. Harrison and Charles Lawrence for many valuable suggestions. This work was partially supported by the Office of Naval Research under contracts ONR N000141613168 and ONR N000141512267.

\section{Authors' contributions}

All authors designed the study. GZ collected the data and performed the statistical analysis. All authors interpreted the data. GZ and SG wrote and revised the paper. All authors read and approved the final manuscript.

\section{Funding}

SG was funded by grants ONR N000141613168 and ONR N000141512267 from the Office of Naval Research. The funding body did not play any role in the design of the study, or collection, analysis, and intepretation of data, or in writing the manuscript.

\section{Availability of Data and Materials}

The dataset analysed during the current study is available at RNA STRAND database [19]. To make the results easily reproducible, a copy of the dataset, as well as code for reproducing the results in the paper, is available at https:// github.com/StannisZhou/rna_statistics.

\section{Ethics Approval and Consent to Participate}

Not applicable.

\section{Consent for Publication}

Not applicable.

\section{Competing Interests}

The authors declare that they have no competing interests.

\section{Author details}

${ }^{1}$ Vicarious AI, Union City, CA USA. ${ }^{2}$ Data Science Institute, Columbia University, New York, NY, USA. ${ }^{3}$ Division of Applied Mathematics, Brown University, Providence, RI, USA.

Received: 6 August 2019 Accepted: 2 December 2019

Published online: 12 December 2019

\section{References}

1. Morris KV, Mattick JS. The rise of regulatory RNA. Nat Rev Genet. 2014;15(6):423-37.

2. Kung JTY, Colognori D, Lee JT. Long noncoding RNAs: past, present, and future. Genetics. 2013;193(3):651-69.

3. Herschlag D. Rna chaperones and the rna folding problem. J Biol Chem. 1995:270(36):20871-4.

4. Pyle AM, Fedorova O, Waldsich C. Folding of group II introns: a model system for large, multidomain RNAs? Trends Biochem Sci. 2007;32(3): 138-45.

5. Zemora G, Waldsich C. Rna folding in living cells. RNA Biol. 2010;7(6): 634-41. https://doi.org/10.4161/rna.7.6.13554. http://arxiv.org/abs/ https://doi.org/10.4161/rna.7.6.13554.

6. Solomatin SV, Greenfeld M, Chu S, Herschlag D. Multiple native states reveal persistent ruggedness of an rna folding landscape. Nature. 2010;463(7281):681.

7. Pyle AM. Group ii intron self-splicing. Ann Rev Biophys. 2016;45(1): 183-205. https://doi.org/10.1146/annurev-biophys-062215-011149. PMID: 27391926

8. Duss O, Stepanyuk GA, Grot A, O'Leary SE, Puglisi JD, Williamson JR. Real-time assembly of ribonucleoprotein complexes on nascent rna transcripts. Nature Commun. 2018;9(1):5087. https://doi.org/10.1038/ s41467-018-07423-3.

9. Lambowitz AM, Perlman PS. Involvement of aminoacyl-tRNA synthetases and other proteins in group I and group II intron splicing. Trends Biochem Sci. 1990;15(11):440-4.

10. Fedorova O, Zingler N. Group II introns: structure, folding and splicing mechanism. Biol Chem. 2007;388(7):665-78. 
11. Chu VB, Herschlag D. Unwinding RNA's secrets: advances in the biology, physics, and modeling of complex RNAs. Curr Opin Struct Biol. 2008;18(3): 305-14.

12. Woodson SA. Taming free energy landscapes with RNA chaperones. RNA Biol. 2010;7(6):677-86

13. Tan Z, Zhang W, Shi $Y$, Wang F. RNA folding: structure prediction, folding kinetics and ion electrostatics. Adv Exp Med Biol. 2015;827:143-83.

14. Leamy KA, Assmann SM, Mathews DH, Bevilacqua PC. Bridging the gap between in vitro and in vivo RNA folding. Q Rev Biophys. 2016;49:10.

15. Chen S-J. RNA folding: conformational statistics, folding kinetics, and ion electrostatics. Annu Rev Biophys. 2008;37:197-214.

16. Lambowitz AM, Caprara MG, Zimmerly S, Perlman PS. Group I and group II ribozymes as RNPs: clues to the past and guides to the future. In: In The RNA World, 2nd. Cold Spring Harbor Laboratory Press; 1999. p. 451-85. http://citeseerx.ist.psu.edu/viewdoc/summary?doi=10.1.1.692.2748.

17. James BD, Olsen GJ, Pace NR. Phylogenetic comparative analysis of RNA secondary structure. Methods Enzymol. 1989;180:227-39.

18. Gutell RR, Lee JC, Cannone JJ. The accuracy of ribosomal RNA comparative structure models. Curr Opin Struct Biol. 2002;12(3):301-10.

19. Andronescu M, Bereg $V$, Hoos HH, Condon A. RNA STRAND: The RNA secondary structure and statistical analysis database. BMC Bioinformatics. 2008;9(1):340.

20. Lorenz R, Bernhart SH, Höner zu Siederdissen C, Tafer H, Flamm C, Stadler PF, Hofacker IL. ViennaRNA package 2.0. Algorithms Mol Biol. 2011;6(1):26.

21. Puton T, Kozlowski LP, Rother KM, Bujnicki JM. CompaRNA: a server for continuous benchmarking of automated methods for RNA secondary structure prediction. Nucleic Acids Res. 2013;41(7):4307-23.

22. Mathews DH. How to benchmark RNA secondary structure prediction accuracy. Methods. 2019;162-163:60-7.

23. Mathews DH, Sabina J, Zuker M, Turner DH. Expanded sequence dependence of thermodynamic parameters improves prediction of RNA secondary structure. J Mol Biol. 1999;288(5):911-40.

24. Zuker M, Mathews DH, Turner DH. Algorithms and thermodynamics for RNA secondary structure prediction: A practical guide. In: Barciszewski J, Clark BFC, editors. RNA Biochem Biotechnol. Dordrecht: Springer; 1999. p. $11-43$.

25. Levinthal C. How to fold graciously. Mossbauer spectroscopy in biological systems. 1969;67:22-4.

26. Higgs PG. RNA secondary structure: physical and computational aspects. Q Rev Biophys. 2000;33(3):199-253.

27. Flamm C, Hofacker IL. Beyond energy minimization: approaches to the kinetic folding of RNA. Monatsh Chem. 2008;139(4):447-57.

28. Baker D, Agard DA. Kinetics versus thermodynamics in protein folding. Biochemistry. 1994;33(24):7505-9.

29. Morgan SR, Higgs PG. Evidence for kinetic effects in the folding of large RNA molecules. J Chem Phys. 1996;105(16):7152-7.

30. Pörschke D. Model calculations on the kinetics of oligonucleotide double helix coil transitions. evidence for a fast chain sliding reaction. Biophys Chem. 1974;2(2):83-96.

31. Pörschke D. A direct measurement of the unzippering rate of a nucleic acid double helix. Biophys Chem. 1974;2(2):97-101.

32. Pörschke D. Elementary steps of base recognition and helix-coil transitions in nucleic acids. Mol Biol Biochem Biophys. 1977;24:191-218.

33. Mohan S, Hsiao C, VanDeusen H, Gallagher R, Krohn E, Kalahar B, Wartell RM, Williams LD. Mechanism of RNA double Helix-Propagation at atomic resolution. J Phys Chem B. 2009;113(9):2614-23.

34. Kandel $D$, Matias $Y$, Unger R, Winkler P. Shuffling biological sequences. Discrete Appl Math. 1996;71(1):171-85.

35. Fitch WM. Random sequences. J Mol Biol. 1983;163(2):171-6.

36. Altschul SF, Erickson BW. Significance of nucleotide sequence alignments: a method for random sequence permutation that preserves dinucleotide and codon usage. Mol Biol Evol. 1985;2(6):526-38.

37. Smit S, Rother K, Heringa J, Knight R. From knotted to nested RNA structures: a variety of computational methods for pseudoknot removal. RNA. 2008;14(3):410-6.

38. Ding $Y$, Lawrence CE. A statistical sampling algorithm for RNA secondary structure prediction. Nucleic Acids Res. 2003;31(24):7280-301.

39. Mathews DH. Revolutions in RNA secondary structure prediction. J Mol Biol. 2006;359(3):526-32.
40. Ding $Y$, Chan $C Y$, Lawrence $C E$. RNA secondary structure prediction by centroids in a boltzmann weighted ensemble. RNA. 2005;11(8):1157-66.

41. Lin L, McKerrow WH, Richards B, Phonsom C, Lawrence CE. Characterization and visualization of RNA secondary structure boltzmann ensemble via information theory. BMC Bioinformatics. 2018;19(1):82.

42. Lai D, Proctor JR, Meyer IM. On the importance of cotranscriptional rna structure formation. RNA. 2013;19(11):1461-73. https://doi.org/10.1261/ rna.037390.112. http://arxiv.org/abs/http://rnajournal.cshlp.org/content/ 19/11/1461.full.pdf+html.

43. Cannone JJ, Subramanian S, Schnare MN, Collett JR, D'Souza LM, Du Y, Feng B, Lin N, Madabusi LV, Müller KM, Pande N, Shang Z, Yu N, Gutell RR. The comparative RNA web (CRW) site: an online database of comparative sequence and structure information for ribosomal, intron, and other RNAs. BMC Bioinformatics. 2002;3:2.

44. Andersen ES, Rosenblad MA, Larsen N, Westergaard JC, Burks J, Wower IK, Wower J, Gorodkin J, Samuelsson T, Zwieb C. The tmRDB and SRPDB resources. Nucleic Acids Res. 2006;34(Database issue):163-8.

45. Brown JW. The ribonuclease P database. Nucleic Acids Res. 1999;27(1):314

46. Gutell RR, Power A, Hertz GZ, Putz EJ, Stormo GD. Identifying constraints on the higher-order structure of RNA: continued development and application of comparative sequence analysis methods. Nucleic Acids Res. 1992;20(21):5785-95.

47. Markham NR, Zuker M. UNAFold: software for nucleic acid folding and hybridization. Methods Mol Biol. 2008;453:3-31.

48. Reuter JS, Mathews DH. RNAstructure: software for RNA secondary structure prediction and analysis. BMC Bioinformatics. 2010;11:129.

49. Zadeh JN, Steenberg CD, Bois JS, Wolfe BR, Pierce MB, Khan AR, Dirks RM, Pierce NA. NUPACK: Analysis and design of nucleic acid systems. J Comput Chem. 2011;32(1):170-3.

50. Hamada M, Kiryu H, Sato K, Mituyama T, Asai K. Prediction of RNA secondary structure using generalized centroid estimators. Bioinformatics. 2009;25(4):465-73.

51. Reeder J, Giegerich R. RNA secondary structure analysis using the RNAshapes package. Curr Protoc Bioinforma. 2009; Chapter 12:12-8.

52. Jiang M, Anderson J, Gillespie J, Mayne M. ushuffle: a useful tool for shuffling biological sequences while preserving the k-let counts. BMC Bioinformatics. 2008;9:192.

\section{Publisher's Note}

Springer Nature remains neutral with regard to jurisdictional claims in published maps and institutional affiliations.
Ready to submit your research? Choose BMC and benefit from:

- fast, convenient online submission

- thorough peer review by experienced researchers in your field

- rapid publication on acceptance

- support for research data, including large and complex data types

- gold Open Access which fosters wider collaboration and increased citations

- maximum visibility for your research: over $100 \mathrm{M}$ website views per year

At BMC, research is always in progress.

Learn more biomedcentral.com/submissions 